\title{
MODELAGEM DO ELO DE CORRENTE CONTÍNUA NO DOMÍNIO DA FREQUÊNCIA EM SISTEMAS ASSÍNCRONOS DESEQUILIBRADOS
}

\author{
Filipe Magno Mayrink Niquini* \\ magno.elt@gmail.com \\ José Luiz R. Pereira* \\ jluiz@ieee.org
}

\author{
Abilio Manuel Variz* \\ abilio@ieee.org \\ Pedro G. Barbosa* \\ pedro.gomes@ufjf.edu.br
}

\author{
Sandoval Carneiro Jr. ${ }^{\dagger}$ \\ sandovaldcoep.ufrj.br \\ *PPEE-UFJF \\ Programa de Pós-Graduação em Engenharia Elétrica \\ Universidade Federal de Juiz de Fora \\ Juiz de Fora, MG, CEP 36015-400, Brasil \\ ${ }^{\dagger}$ COPPE-UFRJ \\ Programa de Engenharia Elétrica \\ Universidade Federal do Rio de Janeiro \\ Caixa Postal 68504 \\ Rio de Janeiro, RJ, 21945-970, Brasil
}

\section{RESUMO}

Este trabalho propõe a modelagem de Elos de Corrente Contínua (HVDC, High Voltage Direct Current Link) para análise harmônica em sistemas elétricos de potência através de uma formulação no domínio da freqüência com representação completa e explícita dos sistemas CA e CC, bem como das características não-lineares dos conversores de potência. O desenvolvimento do modelo proposto é baseado no Método de Injeção de Correntes Trifásico Harmônico (MICTH) e permite a simulação sistêmica de redes CA com elo CC de alta complexidade, como sistemas com conversores de 12 pulsos e assíncronos. Os resultados das simulações do modelo proposto, no domínio da frequiência, foram validados a partir de comparações com resultados obtidos pelo ATP (Alternative Transients Program) no domínio do tempo.

Artigo submetido em 16/03/2009 (Id.: 00972)

Revisado em 06/05/2009, 15/07/2009

Aceito sob recomendação do Editor Associado Prof. Antonio Carlos Zambroni de Souza
PALAVRAS-CHAVE: Análise e Simulação Harmônica, Elo de Corrente Contínua de 12 pulsos (HVDC), Fluxo de Potência Harmônico, Interconexões Assíncronas, Propagação Harmônica.

\section{ABSTRACT}

HVDC Modeling in Frequency Domain for Harmonic Analysis of Unbalanced Asynchronous Systems

This work proposes the modeling of High Voltage Direct Current Link (HVDC) for harmonic analysis in AC electrical power systems using frequency domain formulation with complete and explicit representation of the AC and DC networks, as well as, the converters non-linear characteristics and their harmonic interdependences. The proposed model development is based on the Three-phase Harmonic Current Injection Method (THCIM) and it allows the systemic simulation of AC networks with DC link of high complexity, as 12-pulse converter systems and asynchronous AC connection. The proposed model simulation results, in frequency 
domain, were validated against ATP (Alternative Transients Program) results, in time domain simulation.

KEYWORDS: Harmonic Load Flow Analysis, Harmonic Analysis and Simulation, 12 Pulse HVDC Link, Power System Harmonics Propagation, Asynchronous Interconnections.

\section{INTRODUÇÃO}

A energia elétrica é fundamental para desenvolvimento da sociedade moderna e está presente em praticamente todas as atividades humanas. Alternativas para sua produção têm sido foco de muitos estudos e investimentos. Porém, exigências como qualidade e confiabilidade da energia entregue (Madrigal, 2001), bem como restrições ambientais, são questões de suma importância em qualquer estudo de sistemas elétricos de potência. Neste contexto, a interconexão de sistemas de energia tem se mostrado cada vez mais atrativa, dado que excedentes de energia de uma dada região podem, por exemplo, assegurar o suprimento de outras regiões em déficit, além de garantir uma maior confiabilidade do sistema como um todo e de possibilitar um melhor despacho. Essa tem se mostrado uma tendência global, e não apenas para sistemas locais, dentro de um mesmo país, mas também como uma integração internacional. Um bom exemplo disso é o Brasil, com exceção de apenas pequenas regiões no norte (como a cidade de Manaus), apresenta um extenso sistema interligado, com dimensões continentais. Apenas 3,4\% da capacidade de produção de eletricidade do país se encontram fora do SIN (Sistema Interligado Nacional) (ONS, 2007). Além da ampla interconexão nacional, existem ainda dois grandes projetos internacionais, o de Garabi, através de uma ligação back-toback com a Argentina, totalizando a importação de $2000 \mathrm{MW}$ (Graham et alli, 2002) e o mais famoso, o de Itaipu binacional (Praça et alli, 1996), atendendo ao Brasil e ao Paraguai. Esse último corresponde a uma planta de cerca de 14000 MW, sendo quase a totalidade destinada ao Brasil. Ambos os projetos, por relacionarem países que operam em frequiências distintas (o Brasil com $60 \mathrm{~Hz}$ e a Argentina e o Paraguai com $50 \mathrm{~Hz}$ ), são interconectados através de Elos de Corrente Contínua (HVDC, High Voltage Direct Current Link). Esse último projeto constitui um dos maiores destaques mundial na operação de sistemas HVDC, aliando uma grande capacidade de produção e transmissão de energia, com capacidade de transferência de $6300 \mathrm{MW}$ em corrente contínua (para o sistema sudeste do Brasil) e a maior tensão CC associada, 600 kV (IEEE, 2006; Angelidis et alli, 2006).

A utilização de sistemas de corrente contínua para a interligação de sistemas somente é imperativa nos casos de interconexões assíncronas, ou seja, para junção de sistemas que operam em freqüências nominais distintas. Porém, ainda assim existem diversas situações em que a sua utilização resulta em benefícios operativos e econômicos, como a transmissão submarina, inviável em CA até mesmo para pequenas distâncias, (cerca de $40 \mathrm{~km}$ ) (Kundur, 1994), ou para a transmissão a longas distâncias (geralmente superiores a $600 \mathrm{~km}$ ), onde a ausência de compensações ao longo da linha é bastante atrativa.

Devido às características não-lineares da operação dos conversores e à alta capacidade de transmissão de potência, os sistemas HVDC geram componentes harmônicos de corrente, cuja propagação pelo sistema resulta em distorções consideráveis nas formas de onda da tensão em vários pontos da rede (Bathurst et alli, 1999). Estas distorções harmônicas podem causar diversos efeitos indesejáveis, como perda de eficiência, interferências em sistemas de comunicação, distúrbios em equipamentos eletrônicos e ressonâncias (IEEE, 1993). A análise de redes em situações em que se deseja obter informações precisas sobre o nível e a localização das distorções harmônicas, geralmente é realizada através de simulações no domínio do tempo, com programas como o EMTP (Electromagnetic Transients Program) (Dommel, 1986), o PSCAD/EMTDC ou o PSB/Simulink (Faruque et alli, 2006). Porém, o esforço computacional associado à solução de redes completas no domínio do tempo é, em geral, demasiadamente elevado dada a necessidade de se simular todo o período transitório antes de se atingir o regime estacionário (Smith et alli, 1995). Por outro lado, simulações com modelos no domínio da freqüência para análise harmônica são geralmente deficientes, devido à grande complexidade envolvida na modelagem de elementos não-lineares, principalmente os conversores de potência (IEEE, 1993 e 1996). Esses são representados quase sempre com diversas simplificações, e muitas vezes, como forma de garantir uma solução mais precisa, utilizam modelos híbridos, com etapas no domínio do tempo. Esse tipo de metodologia tem, porém, o inconveniente de não possuir expressões diferenciáveis, o que geralmente exige a utilização de métodos de solução de Gauss-Seidel, comumente passíveis de problemas de convergência (Bathurst et alli, 1999). Nesses casos costuma-se utilizar algum outro método de convergência associado, como forma de melhorar o condicionamento do sistema.

Neste trabalho, as limitações comuns às simulações de redes com sistema HVDC no domínio da freqüência são prontamente superadas, sendo possível uma análise completa e de forma direta, inclusive no que diz respeito às distorções e propagações harmônicas (Niquini et alli, 2008a, 2008b e 2009a). Outra importante contribuição do presente trabalho é a possibilidade de realizar simulações e análises de sistemas assíncronos com conversores de 12 pulsos através de uma metodologia totalmente fundamentada no domínio harmônico, sem qualquer simulação no domínio do tempo. Conforme será abordado, o método proposto permite que interconexões assíncronas, back-to-back ou não, equilibrados 


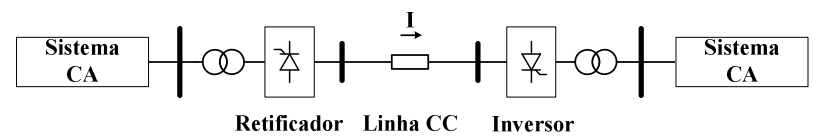

Figura 1: Diagrama Unifilar simplificado de um HVDC.

ou desequilibrados, com distorções harmônicas ou não, possam ser diretamente inseridas no fluxo de potência harmônico, através de uma metodologia robusta e completa.

\section{INTERCONEXÕES DE SISTEMAS CC- CA}

A utilização de sistemas de corrente contínua para a interligação de redes apresenta interessantes características, como a possibilidade de se conectar sistemas de frequiências distintas, a isolação de faltas, o desacoplamento angular de sistemas remotos e o total controle do fluxo de potência ativa pelo elo.

A maioria dos sistemas HVDC existentes hoje utiliza tiristores como elementos de conversão (Rudervall et alli, 2000), o que implica em frequiências de chaveamento iguais à frequiência da rede $\mathrm{CA}$, resultando em componentes harmônicos de baixa ordem (Niquini et alli, 2008a e 2008b). Esses sistemas são chamados de CSC-HVDC (Current Source Converter HVDC, Conversores Fonte de Corrente), ou HVDC clássicos. Atualmente tem se difundido a utilização de chaves autocomutadas em sistemas HVDC, o que tem permitido o desenvolvimento de sistemas mais eficientes, com operação em altas frequiências. Isso resulta numa maior facilidade para controlar as distorções geradas. Esses sistemas são chamados de VSC-HVDC (Voltage Source Converter HVDC, Conversores Fonte de Tensão) (Sood, 2004). Entretanto, ainda assim os CSC-HVDC merecem destaque, e têm como principais atrativos a vantagem de suportarem uma maior tensão de operação e uma maior capacidade de transferência de potência. No presente trabalho, o CSC-HVDC será simplesmente denominado de HVDC. A Figura 1 mostra o diagrama unifilar simplificado de um sistema HVDC. Basicamente, os sistemas HVDC são formados pelas estações conversoras (o retificador e o inversor), pelos transformadores de conexão, responsáveis pelo controle da tensão de entrada e de saída (e para a compatibilização angular em sistemas multi-pulsos) e pela linha CC (exceto em sistemas back-to-back).

A utilização de sistemas HVDC ocorre principalmente em três situações:

- para a conexão de sistemas remotos, onde a viabilidade da linha de transmissão em corrente contínua supera à da linha em corrente alternada;
- para a conexão remota de sistemas com frequiências nominais distintas;

- e para a conexão direta de sistemas assíncronos, sem a necessidade de construção de transmissão em corrente contínua, chamada de back-to-back.

Dentre essas, seguramente a mais complexa refere-se ao acoplamento de sistemas assíncronos, dada a possibilidade de interação entre os sistemas, conforme será discutido mais a frente.

A operação do elo de CC, mesmo sob situações ideais, provoca a geração de componentes harmônicos por parte dos conversores. Os harmônicos produzidos sob condições ideais de funcionamento são chamados de harmônicos característicos (Kundur, 1994). Do lado CA, tanto do retificador quanto do inversor, os harmônicos de corrente possuem componentes $p n \pm 1 \mathrm{com}$ amplitudes $I_{1} / h$, onde $n$ é inteiro positivo, $p$ o número de pulsos do conversor, $h$ a ordem do harmônico em questão e $I_{1}$ a amplitude da componente fundamental. Já nos terminais CC é produzida uma onda de tensão e corrente com espectro harmônico pn cujas amplitudes dependem diretamente do ângulo de disparo $(\alpha)$ dos tiristores. Isso mostra que quanto maior o número de pulsos dos conversores, menores são as distorções harmônicas introduzidas no sistema.

\subsection{Interconexões Assíncronas}

Interconexões assíncronas em sistemas de potência, como já abordado, são as únicas formas onde a utilização de elos CC é fundamental, visto que a conexão direta de redes com freqüências distintas é operacionalmente impossível. A operação de sistemas HVDC nesses casos é notadamente complexa e de difícil representação matemática em modelagens no domínio da freqüência, visto que o desacoplamento entre as redes através da utilização de corrente contínua não é perfeito. Como resultado, tem-se que os componentes harmônicos provenientes de um dos sistemas CA podem se propagar para o outro, se apresentando para este como um componente interharmônico, uma vez que as frequiências, não são múltiplas entre si $(50$ e $60 \mathrm{~Hz})$. Operacionalmente reduzse essa influência através do aumento do reator de alisamento presente entre os conversores, reduzindo os componentes harmônicos (ripple) da corrente CC (CSC-HVDC). Ainda assim não é possível eliminar por completo a interação entre os sistemas conectados ao HVDC, o que traz a necessidade de uma análise mais completa. A modelagem proposta permite superar com êxito todas essas limitações encontradas em metodologias baseadas no domínio da freqüência, garantindo que o sistema possa ser amplamente avaliado, sem qualquer perda de precisão e sem a necessidade de simulações no domínio do tempo. 


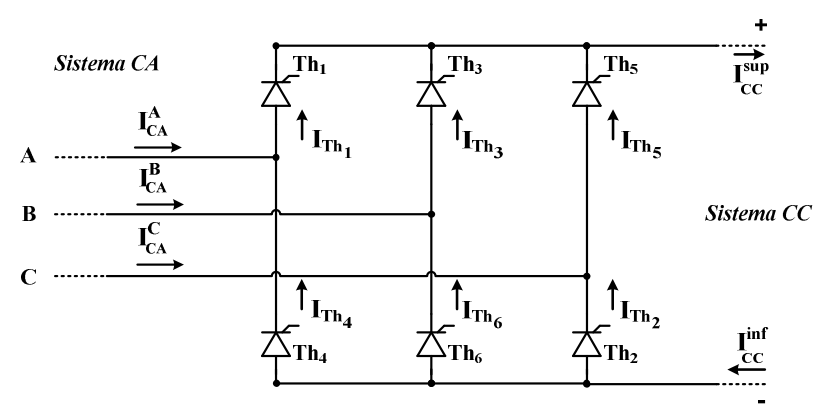

Figura 2: Conversor base, formado por 6 Tiristores ligados em Ponte.

\section{MODELO MATEMÁTICO PROPOSTO}

O modelo desenvolvido para os conversores do HVDC é baseado na ponte de 6 tiristores, conforme mostrado na Figura 2. Na prática, devido às altas tensões e altas correntes envolvidas, vários tiristores são associados em série e em paralelo, formando válvulas. Esquematicamente, porém, esta associação será representada como um único elemento, sendo então as denominações tiristores e válvulas utilizadas como sinônimos no presente trabalho. Nos casos em que apenas uma ponte dessas for utilizada, o conversor é chamado de conversor de 6 pulsos, dado a forma resultante da tensão do lado CC. Para reduzir o efeito dos harmônicos no sistema é feito a associação de duas ou mais pontes, conectadas em série do lado CC. Conseqüentemente os componentes harmônicos são deslocados para ordens mais elevadas, resultando em menores distorções. A configuração mais comumente utilizada é a de 12 pulsos (com duas pontes conectadas). Para tanto, basta apenas que as tensões do lado CA estejam corretamente defasadas, conseguidas através do uso de transformadores, como será explicado mais à frente. Os tiristores são numerados segundo a seqüência natural de condução, lembrando que sempre existem pelo menos duas válvulas conduzindo simultaneamente, uma na parte superior e outra na parte inferior.

A formulação matemática utilizada é fundamentada nas equações de injeção de corrente (Variz, 2006 e 2008), o que garante uma formulação simples e robusta.

\subsection{Fluxo de Potência Trifásico Harmô- nico}

O Fluxo de Potência Trifásico e Harmônico utilizado no presente trabalho é baseado no MICTH (Método de Injeção de Correntes Trifásico Harmônico) originalmente desenvolvido por Variz (2006). A sua formulação é integralmente no domínio da freqüência com solução iterativa baseada no método de Newton-Raphson. O MICTH constitui uma ferramenta de análise harmônica eficiente, robusta e precisa, que permite a simulação da rede completa, ao representar todas as características dos componentes do sistema (Variz et alli, 2008).

O método de Newton, utilizado na resolução do sistema, converge quadraticamente, e geralmente necessita de apenas uma inicialização apropriada das variáveis. Nesse trabalho, apesar da existência de diversas variáveis (da ordem de $6 \cdot \mathrm{Ht} \cdot \mathrm{Nb}$, onde Ht é o número total de freqüências harmônicas simuladas e $\mathrm{Nb}$ o número de barras do sistema) verificase que apenas a inicialização da freqüência fundamental é suficiente para alcançar uma convergência rápida e correta. Nenhuma inicialização dos componentes harmônicos, de nenhuma barra, é necessária para a convergência, nem mesmo para as simulações assíncronas, o que demonstra a grande robustez e versatilidade da metodologia proposta.

O sistema matricial do MICTH é apresentado de forma compacta em (1). Essa matriz é formada pelos subsistemas de todas as freqüências harmônicas simuladas $(h=$ $0,1,2, \ldots, H)$, onde os blocos fora da diagonal principal da matriz Jacobiana representam os acoplamentos harmônicos entre freqüências distintas, necessários para a representação dos elementos não-lineares. Esses acoplamentos permitem que a operação dos conversores leve em consideração todo o conteúdo harmônico do sistema, inclusive as distorções provocadas por eles próprios.

A definição dos harmônicos que serão simulados é totalmente flexível, podendo-se incluir ou excluir qualquer componente desejado, o que contribui para uma representação mais versátil e completa do sistema. Isso torna possível, por exemplo, a exclusão dos harmônicos que, numa dada situação em particular, são inexistentes, reduzindo as dimensões dessa matriz, apesar disso não ser necessário na maioria dos casos.

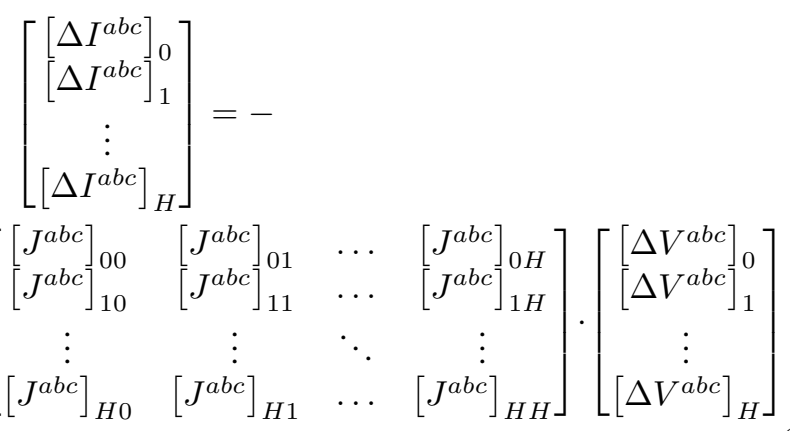

onde:

$\left[\Delta I_{k}^{a b c}\right]_{h}$ é o vetor com os resíduos de corrente da barra $\mathrm{k}$ na freqüência harmônica $h$; 
$\left[J_{k i}^{a b c}\right]_{h h}$ é o bloco $k i$ trifásico complexo da matriz Jacobiana no subsistema de freqüência harmônica $h$;

$\left[J_{k i}^{a b c}\right]_{h n}$ é o bloco $k i$ trifásico complexo da matriz Jacobiana representando os acoplamentos harmônicos entre as frequiências harmônicas $h$ e $n$, devido as nãolinearidades do sistema;

$\left[\Delta V_{k}^{a b c}\right]_{h}$ é o vetor trifásico complexo com os incrementos de tensão nodal da barra $k$ na freqüência harmônica $h$.

Cada um dos blocos presentes na matriz Jacobiana é formado pelas contribuições cumulativas de cada um dos elementos do sistema (como cargas, linhas de transmissão, transformadores, etc.) e são obtidas através das derivadas parciais das injeções de corrente de cada um destes elementos em relação às tensões. O sistema matricial é então formado por componentes trifásicos e complexos, conforme ilustrado em (2) pela expansão do vetor de resíduos na frequiência $h$, onde os subíndices $r$ e $m$ denotam as componentes real e imaginário, respectivamente.

$$
\begin{aligned}
& {\left[\Delta I^{a b c}\right]_{h}=\left[\begin{array}{c}
{\left[\Delta I_{1}^{a b c}\right]} \\
{\left[\Delta I_{2}^{a b c}\right]} \\
\vdots \\
{\left[\Delta I_{N b}^{a b c}\right]}
\end{array}\right]_{h}=}
\end{aligned}
$$

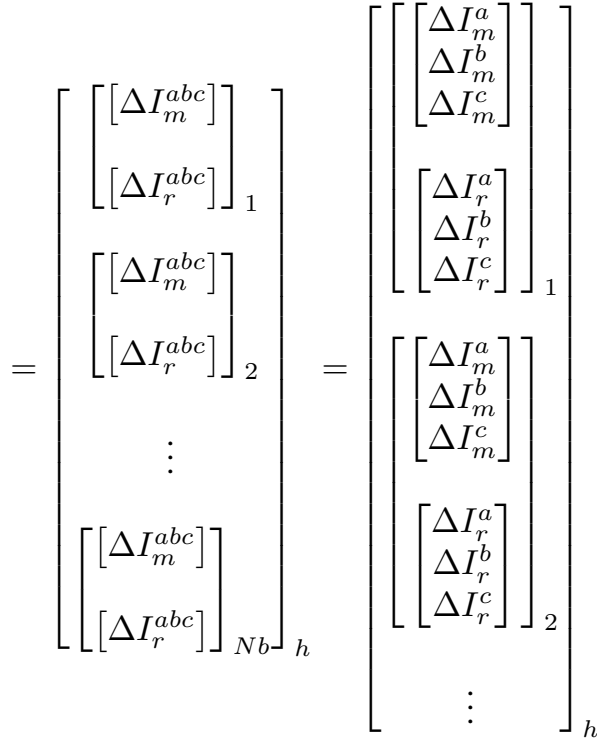

\subsection{Elo de Corrente Contínua: Modela- gem dos Conversores}

Os conversores de potência (retificadores e inversores) presentes nos elos de corrente continua, são equipamentos extremamente complexos de serem modelados no domínio da

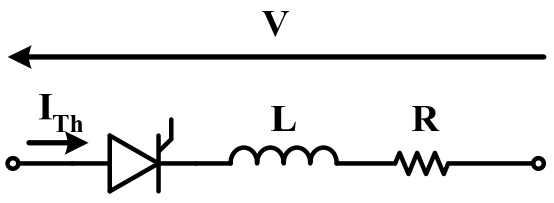

Figura 3: Modelo representativo das Válvulas.

frequiência, devido às suas não-linearidades e à dependência do conteúdo harmônico em seus terminais. Conforme será explorado, mesmo para o cálculo das respostas nas ordens harmônicas mais baixas, o conhecimento da informação harmônica de ordens superiores é primordial.

Para a modelagem dos conversores, cada uma das válvulas é analisada separadamente, segundo a Figura 3. Os parâmetros $R$ e $L$ são, respectivamente, a resistência representativa das perdas do componente e a indutância de proteção contra variações bruscas na corrente durante o ligamento das chaves (turn-on snubber). Note que esses parâmetros não foram representados na Figura 2.

A modelagem tem como ponto de partida, a tensão sobre cada uma das válvulas, dadas pelos coeficientes da série de Fourier, e dos instantes de disparo e corte. Com isso, calculam-se as injeções de correntes para cada uma das válvulas e posteriormente para o conversor, segundo apresentado a seguir (Niquini et alli, 2009b).

Seja então a tensão nos terminais de uma dada válvula expressa pela série de Fourier, dada por (3).

$$
v(t)=V_{0}+\sum_{h=1}^{\infty}\left(V_{r_{h}} \cos (h \omega t)+V_{m_{h}} \operatorname{sen}(h \omega t)\right)
$$

onde:

$V_{0}$ é a componente contínua da tensão;

$V_{r_{h}}$ e $V_{m_{h}}$ são as componentes real e imaginária da tensão na freqüência harmônica $h$;

$\omega$ a freqüência angular fundamental do sistema.

Do modelo representado na Figura 3, tem-se, para uma determinada válvula (Th), a equação diferencial dada em (4).

$$
L \cdot \frac{d I_{T h}(t)}{d t}+R \cdot I_{T h}(t)=v(t)
$$

Aplicando a Transformada de Fourier à solução dessa equação diferencial considerando-se os instantes de disparo e 
corte do tiristor obtêm-se as expressões (5) a (7), que representam, respectivamente, as partes contínua, real e imaginária da corrente na válvula.

$$
\begin{gathered}
I_{0}=\frac{\omega}{2 \pi}\left(I_{0}^{\prime}\left(t_{o f f}\right)-I_{0}^{\prime}\left(t_{\text {on }}\right)\right) \\
I_{r_{h}}=\frac{\omega}{\pi}\left(I_{r}^{\prime}\left(h, t_{o f f}\right)-I_{r}^{\prime}\left(h, t_{o n}\right)\right) \\
I_{m_{h}}=\frac{\omega}{\pi}\left(I_{m}^{\prime}\left(h, t_{o f f}\right)-I_{m}^{\prime}\left(h, t_{o n}\right)\right)
\end{gathered}
$$

Cada um de seus termos, em função da ordem harmônica e dos instantes de disparo ou corte, é dado a seguir pelas expressões (8) a (10).

$$
\begin{gathered}
I_{0}^{\prime}\left(t_{j}\right)=\left(\frac{V_{0} \cdot t_{j}}{R}\right)+\sum_{n=1}^{\infty}\left[+\frac{V_{r_{n}}}{n \omega Z_{n}}\left(\operatorname{sen}\left(n \omega t_{j}-\varphi_{n}\right)\right)\right]+ \\
-\frac{K \cdot L \cdot e^{-\frac{R}{L}\left(t_{j}-t_{o n_{j}}\right)}}{R}+\sum_{n=1}^{\infty}\left[-\frac{V_{m_{n}}}{n \omega Z_{n}}\left(\cos \left(n \omega t_{j}-\varphi_{n}\right)\right)\right]
\end{gathered}
$$

$$
\begin{gathered}
I_{r}^{\prime}\left(h, t_{j}\right)=\left(\frac{V_{0} \operatorname{sen}\left(h \omega t_{j}\right)}{h \omega R}\right)+ \\
\sum_{\substack{n=1 \\
n \neq h}}^{\infty}\left[+\frac{V_{r_{n}}}{2 \omega Z_{n}}\left(\frac{\operatorname{sen}\left((h+n) \omega t_{j}-\varphi_{n}\right)}{h+n}+\right.\right. \\
\left.\left.\quad \frac{\operatorname{sen}\left((h-n) \omega t_{j}+\varphi_{n}\right)}{h-n}\right)\right]+ \\
\sum_{\substack{n=1 \\
n \neq h}}^{\infty}\left[-\frac{V_{m_{n}}}{2 \omega Z_{n}}\left(\frac{\cos \left((n-h) \omega t_{j}-\varphi_{n}\right)}{n-h}+\right.\right. \\
\left.\left.\frac{\cos \left((h+n) \omega t_{j}-\varphi_{n}\right)}{h+n}\right)\right]+ \\
\frac{V_{r_{h}}}{2 \omega Z_{h}}\left(\omega t_{j} \cos \left(\varphi_{h}\right)+\frac{\operatorname{sen}\left(2 h \omega t_{j}-\varphi_{h}\right)}{2 h}\right)- \\
\frac{V_{m_{h}}}{2 \omega Z_{h}}\left(\omega t_{j} \operatorname{sen}\left(\varphi_{h}\right)+\frac{\cos \left(2 h \omega t_{j}-\varphi_{h}\right)}{2 h}\right)+ \\
\frac{K \cdot L \cdot e^{-\frac{R}{L}\left(t_{j}-t_{o n_{j}}\right)}}{\left(Z_{h}\right)^{2}}\left[-R \cos \left(h \omega t_{j}\right)+h \omega L \operatorname{sen}\left(h \omega t_{j}\right)\right]
\end{gathered}
$$

$$
\begin{gathered}
I_{m}^{\prime}\left(h, t_{j}\right)=\left(-\frac{V_{0} \cos \left(h \omega t_{j}\right)}{h \omega R}\right)+ \\
\sum_{\substack{n=1 \\
n \neq h}}^{\infty}\left[-\frac{V_{r_{n}}}{2 \omega Z_{n}}\left(\frac{\cos \left((h+n) \omega t_{j}-\varphi_{n}\right)}{h+n}+\right.\right. \\
\left.\left.\quad \frac{\cos \left((h-n) \omega t_{j}+\varphi_{n}\right)}{h-n}\right)\right]+ \\
\left.\sum_{\substack{n=1 \\
n \neq h}}^{\infty}\left[+\frac{V_{m_{n}}\left(\frac{\operatorname{sen}\left((n-h) \omega t_{j}-\varphi_{n}\right)}{2 \omega Z_{n}}-\right.}{n-h}\right)\right]+ \\
\left.\left.\frac{\operatorname{sen}\left((h+n) \omega t_{j}-\varphi_{n}\right)}{h+n}\right)\right]+ \\
\frac{V_{r_{h}}}{2 \omega Z_{h}}\left(\omega t_{j} \operatorname{sen}\left(\varphi_{h}\right)-\frac{\cos \left(2 h \omega t_{j}-\varphi_{h}\right)}{2 h}\right)+ \\
\frac{V_{m_{h}}}{2 \omega Z_{h}}\left(\omega t_{j} \cos \left(\varphi_{h}\right)-\frac{\operatorname{sen}\left(2 h \omega t_{j}-\varphi_{h}\right)}{2 h}\right)+ \\
\frac{K \cdot L \cdot e^{-\frac{R}{L}\left(t_{j}-t_{o n_{j}}\right)}}{\left(Z_{h}\right)^{2}}\left[-R \operatorname{sen}\left(h \omega t_{j}\right)-h \omega L \cos \left(h \omega t_{j}\right)\right] \\
\text { onde: } \quad(10)
\end{gathered}
$$

$$
\begin{gathered}
Z_{n}=\sqrt{R^{2}+(n \omega L)^{2}} \\
\varphi_{n}=\operatorname{arctg}\left(\frac{n \omega L}{R}\right) \\
K=-\frac{V_{0}}{R}-\sum_{n=1}^{\infty}\left[\frac { 1 } { Z _ { n } } \left(V_{r_{n}} \cos \left(n \omega t_{j}-\varphi_{n}\right)+\right.\right. \\
\left.\left.V_{m_{n}} \operatorname{sen}\left(n \omega t_{j}-\varphi_{n}\right)\right)\right]
\end{gathered}
$$

sendo:

$t_{j}$ o instante de disparo ou corte dos tiristores.

Desenvolvimento matemático análogo é repetido para cada uma das válvulas do conversor apresentado na Figura 2, obtendo-se desta forma, todas as correntes em todas as válvulas do conversor $\left(I_{T h 1}, I_{T h 2}, \ldots, I_{T h 6}\right)$. Assim, as correntes harmônicas injetadas pelo conversor (retificador ou inversor) em seus terminais CC e CA são calculadas, respectivamente, pelas equações (12) e (13).

$$
\begin{aligned}
& I_{C C}^{\text {sup }}=I_{T h_{1}}+I_{T h_{3}}+I_{T h_{3}} \\
& I_{C C}^{i n f}=I_{T h_{2}}+I_{T h_{4}}+I_{T h_{6}}
\end{aligned}
$$




$$
\begin{aligned}
& I_{C A}^{A}=I_{T h_{1}}-I_{T h_{4}} \\
& I_{C A}^{B}=I_{T h_{3}}-I_{T h_{6}} \\
& I_{C A}^{B}=I_{T h_{5}}-I_{T h_{2}}
\end{aligned}
$$

O instante de disparo de cada tiristor é medido a partir do instante em que a componente fundamental da tensão sobre a válvula se torna positiva, ou seja, a partir do momento em que a válvula fica diretamente polarizada. É possível perceber que esse instante independe da tensão CC e está associado apenas com as tensões $\mathrm{CA}$ na fase referente à válvula que está em condução e com a tensão CA da válvula que entrará em condução em seguida, portanto leva em consideração os desequilíbrios e distorções existentes na rede. Para os instantes de corte é considerado o período ideal de condução, no caso $120^{\circ}$.

As contribuições do conversor na matriz Jacobiana são calculadas através da diferenciação das equações de injeção de correntes (12) e (13) em relação às tensões harmônicas. Portanto são formadas pela soma das derivadas parciais da corrente de cada uma das válvulas obtidas pela diferenciação das equações (5) a (7), que por sua vez são calculadas pelas expressões (8) a (10). Desta forma, as derivadas parciais da componente CC da corrente $(h=0)$ são dadas pelas equações (14) a (16).

$$
\begin{gathered}
\frac{\partial I_{0}^{\prime}(t)}{\partial V_{0}}=\frac{1}{R}\left[t+\frac{L e^{-\frac{R}{L}\left(t-t_{o n}\right)}}{R}\right] \\
\frac{\partial I_{0}^{\prime}(t)}{\partial V_{r_{n}}}=\frac{1}{\omega Z_{n}}\left[\frac{\operatorname{sen}\left(n \omega t-\varphi_{n}\right)}{n}+\right. \\
\left.\omega \cos \left(n \omega t_{o n}-\varphi_{n}\right) \frac{L e^{-\frac{R}{L}\left(t-t_{o n}\right)}}{R}\right] \\
\frac{\partial I_{0}^{\prime}(t)}{\partial V_{m_{n}}}=\frac{1}{\omega Z_{n}}\left[\frac{-\cos \left(n \omega t-\varphi_{n}\right)}{n}+\right. \\
\left.\omega \operatorname{sen}\left(n \omega t_{o n}-\varphi_{n}\right) \frac{L e^{-\frac{R}{L}\left(t-t_{o n}\right)}}{R}\right]
\end{gathered}
$$

As derivadas parciais dos componentes harmônicos de corrente para uma dada ordem harmônica $h$ em relação à tensão CC são apresentadas pelas equações (17) a (18).

$$
\begin{aligned}
& \frac{\partial I_{r}^{\prime}(h, t)}{\partial V_{0}}=\frac{1}{R}\left[\frac{\operatorname{sen}(h \omega t)}{\omega h}+\right. \\
& \left.-\frac{L e^{-\frac{R}{L}\left(t-t_{o n}\right)}}{Z_{h}^{2}}(-R \cos (h \omega t)+h \omega L \operatorname{sen}(h \omega t))\right]
\end{aligned}
$$

$$
\begin{aligned}
& \frac{\partial I_{m}^{\prime}(h, t)}{\partial V_{0}}=\frac{1}{R}\left[-\frac{\cos (h \omega t)}{\omega h}+\right. \\
& \left.-\frac{L e^{-\frac{R}{L}\left(t-t_{o n}\right)}}{Z_{h}^{2}}(-R \operatorname{sen}(h \omega t)-h \omega L \cos (h \omega t))\right]
\end{aligned}
$$

A seguir, nas equações (19) a (22), são apresentadas as derivadas parciais que formam os blocos da diagonal principal da Jacobiana, ou seja, os blocos referentes a apenas uma frequiência harmônica:

$$
\begin{array}{r}
\frac{\partial I_{r}^{\prime}(h, t)}{\partial V_{r_{h}}}=\frac{1}{2 \omega Z_{h}}\left[\omega t \cos \left(\varphi_{h}\right)+\frac{\operatorname{sen}\left(2 h \omega t-\varphi_{h}\right)}{2 h}+\right. \\
-2 \omega \cos \left(h \omega t_{o n}-\varphi_{h}\right) \frac{L e^{-\frac{R}{L}\left(t-t_{o n}\right)}}{Z_{h}^{2}} \\
(-R \cos (h \omega t)+h \omega L \operatorname{sen}(h \omega t))]
\end{array}
$$

$$
\begin{gathered}
\frac{\partial I_{r}^{\prime}(h, t)}{\partial V_{m_{h}}}=\frac{1}{2 \omega Z_{h}}\left[-\omega t \operatorname{sen}\left(\varphi_{h}\right)-\frac{\cos \left(2 h \omega t-\varphi_{h}\right)}{2 h}+\right. \\
-2 \omega \operatorname{sen}\left(h \omega t_{o n}-\varphi_{h}\right) \frac{L e^{-\frac{R}{L}\left(t-t_{o n}\right)}}{Z_{h}^{2}} \\
(-R \cos (h \omega t)+h \omega L \operatorname{sen}(h \omega t))]
\end{gathered}
$$

$$
\begin{gathered}
\frac{\partial I_{m}^{\prime}(h, t)}{\partial V_{r_{h}}}=\frac{1}{2 \omega Z_{h}}\left[\omega t \operatorname{sen}\left(\varphi_{h}\right)-\frac{\cos \left(2 h \omega t-\varphi_{h}\right)}{2 h}+\right. \\
-2 \omega \cos \left(h \omega t_{o n}-\varphi_{h}\right) \frac{L e^{-\frac{R}{L}\left(t-t_{o n}\right)}}{Z_{h}^{2}} \\
(-R \operatorname{sen}(h \omega t)-h \omega L \cos (h \omega t))]
\end{gathered}
$$

$$
\begin{gathered}
\frac{\partial I_{m}^{\prime}(h, t)}{\partial V_{m_{h}}}=\frac{1}{2 \omega Z_{h}}\left[\omega t \cos \left(\varphi_{h}\right)-\frac{\operatorname{sen}\left(2 h \omega t-\varphi_{h}\right)}{2 h}+\right. \\
-2 \omega \operatorname{sen}\left(h \omega t_{o n}-\varphi_{h}\right) \frac{L e^{-\frac{R}{L}\left(t-t_{o n}\right)}}{Z_{h}^{2}} \\
(-R \operatorname{sen}(h \omega t)-h \omega L \cos (h \omega t))]
\end{gathered}
$$


Por fim, as derivadas parciais que constituem os blocos representativos dos acoplamentos harmônicos para as freqüências diferentes de zero são dadas pelas equações (23) a (26). Estes blocos estão situados fora da diagonal principal da matriz Jacobiana.

$$
\begin{gathered}
\frac{\partial I_{r}^{\prime}(h, t)}{\partial V_{r_{n}}}=\frac{1}{2 \omega Z_{n}}\left[\frac{\operatorname{sen}\left((h+n) \omega t-\varphi_{n}\right)}{h+n}+\right. \\
\frac{\operatorname{sen}\left((h-n) \omega t+\varphi_{n}\right)}{h-n}-2 \omega \cos \left(n \omega t_{o n}-\varphi_{n}\right) \frac{L e^{-\frac{R}{L}\left(t-t_{o n}\right)}}{Z_{h}^{2}} \\
(-R \cos (h \omega t)+h \omega L \operatorname{sen}(h \omega t))]
\end{gathered}
$$

$$
\begin{gathered}
\frac{\partial I_{r}^{\prime}(h, t)}{\partial V_{m_{n}}}=-\frac{1}{2 \omega Z_{n}}\left[\frac{\cos \left((n-h) \omega t-\varphi_{n}\right)}{n-h}+\right. \\
\frac{\cos \left((n+h) \omega t-\varphi_{n}\right)}{n+h}+2 \omega \operatorname{sen}\left(n \omega t_{o n}-\varphi_{n}\right) \frac{L e^{-\frac{R}{L}\left(t-t_{o n}\right)}}{Z_{h}^{2}} \\
(-R \cos (h \omega t)+h \omega L \operatorname{sen}(h \omega t))]
\end{gathered}
$$

$$
\begin{gathered}
\frac{\partial I_{m}^{\prime}(h, t)}{\partial V_{r_{n}}}=-\frac{1}{2 \omega Z_{n}}\left[\frac{\cos \left((h+n) \omega t-\varphi_{n}\right)}{h+n}+\right. \\
\frac{\cos \left((h-n) \omega t+\varphi_{n}\right)}{h-n}+2 \omega \cos \left(n \omega t_{o n}-\varphi_{n}\right) \frac{L e^{-\frac{R}{L}\left(t-t_{o n}\right)}}{Z_{h}^{2}} \\
(-R \operatorname{sen}(h \omega t)-h \omega L \cos (h \omega t))]
\end{gathered}
$$

$$
\begin{aligned}
& \frac{\partial I_{m}^{\prime}(h, t)}{\partial V_{m_{n}}}=\frac{1}{2 \omega Z_{n}} {\left[\frac{\operatorname{sen}\left((n-h) \omega t-\varphi_{n}\right)}{h+n}-\right.} \\
& \frac{\operatorname{sen}\left((n+h) \omega t-\varphi_{n}\right)}{n+h}-2 \omega \operatorname{sen}\left(n \omega t_{o n}-\varphi_{n}\right) \frac{L e^{-\frac{R}{L}\left(t-t_{o n}\right)}}{Z_{h}^{2}} \\
&(-R \operatorname{sen}(h \omega t)-h \omega L \cos (h \omega t))]
\end{aligned}
$$

\subsubsection{Sistema de 12 Pulsos}

Sistemas de 12 pulsos podem ser conseguidos pela associação de dois conversores de 6 pulsos, conectados em série do lado CC. Essa associação permite a construção de três sistemas distintos: (i) um consistindo de um sistema monopolar, onde existe somente um caminho para a circulação de corrente; (ii) outro formando um sistema homopolar, ou seja, um sistema em que ambas as linhas de transmissão CC tem o mesmo potencial, geralmente o negativo, (iii) e outro formando um sistema bipolar, com um dos condutores

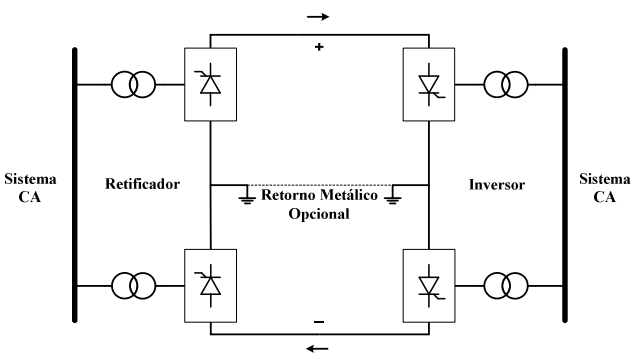

Figura 4: Elo de Corrente Contínua Bipolar.

com polaridade positiva e outro com a polaridade negativa. As junções entre os conversores de 6 pulsos desses dois últimos tipos são aterradas, como em uma fonte simétrica, formando então dois pólos (dois caminhos para a passagem da corrente). Nesses sistemas o fluxo de potência pode ser facilmente invertido, sem a necessidade de chaveamentos mecânicos. A maioria dos sistemas HVDC opera na forma bipolar, passando à operação monopolar no caso de contingências (Kundur, 1994). O diagrama unifilar desse modelo é apresentado na Figura 4.

As diferenças na implementação desse sistema em relação ao sistema simples de 6 pulsos (Niquini et alli, 2008a, 2008b e 2009a) residem na contribuição cumulativa de cada um dos conversores nas barras comuns a ambos os pólos e a necessidade de utilização de transformadores adequados para a realização da defasagem das tensões de entrada e saída dos conversores. Todos os demais cálculos são realizados da mesma forma. Note que as injeções de corrente do conversor no nó comum são calculadas então pela soma das injeções dos dois conversores base. Os transformadores utilizados nesses sistemas desempenham duas funções, a de controlar o nível de tensão CA de entrada no retificador e a de saída no inversor (permitindo um amplo controle do fluxo de potência no sistema), e a de promover o defasamento angular necessário para o funcionamento dos conversores. No caso dos sistemas de 6 pulsos, cada válvula conduz por $120^{\circ}$, mas a cada $60^{\circ}$ ocorre a comutação de uma válvula para outra. No caso de sistemas de 12 pulsos, cada válvula continua conduzindo da mesma forma, caso seja analisado cada um dos pólos separadamente, porém, ao analisar o sistema completo tem-se a comutação de válvulas a cada $30^{\circ}$. Para que isso ocorra, ou seja, para que o sistema como um todo resulte nos 12 pulsos pretendidos, é preciso apenas que ambos os pólos estejam defasados entre si de $30^{\circ}$. Essa diferença angular é conseguida através da utilização de transformadores conectados em estrela-aterrada/estrela em um dos pólos e em estrela-aterrada/delta em outro (Kundur, 1994), tanto do lado retificador quanto do lado inversor.

O retorno existente entre os conversores somente é necessário em casos de desequilíbrios de operação ou na situação 


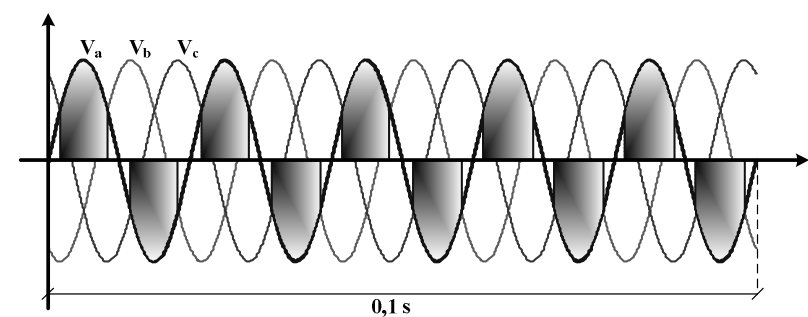

Figura 5: Períodos de Condução para as Válvulas do Lado de $50 \mathrm{~Hz}$.

de uma falta em uma das linhas CC, onde o sistema poderia passar a operar, temporariamente, de forma monopolar, com a utilização desse retorno. Apenas são construídos retornos metálicos nas situações em que a circulação de correntes por terra ou água (no caso de travessias submarinas) possa comprometer alguma estrutura já existente (Kundur, 1994).

\subsubsection{Interconexões Assíncronas}

A simulação de sistemas assíncronos pode ser encarada como um grande desafio para as metodologias no domínio da freqüência, especialmente pelo fato das freqüências nominais de cada sistema CA não serem múltiplas inteiras uma da outra. Nesse caso, as equações anteriormente desenvolvidas não podem ser diretamente utilizadas, dado que é necessária a utilização de um período fundamental comum para todo o sistema, que neste caso não se pode estar associado nem a 50 nem $60 \mathrm{~Hz}$. Assim, em essência, a estratégia adotada baseiase na utilização de um período fundamental que contemple ambas as freqüências como múltiplas inteiras. No caso, a freqüência a ser utilizada é a de $10 \mathrm{~Hz}$ (máximo divisor comum entre 50 e 60). Assim, durante o período correspondente à freqüência de $10 \mathrm{~Hz}$, todos os instantes de condução de cada uma das válvulas são considerados. Dessa forma, as válvulas do lado de $50 \mathrm{~Hz}$ conduzirão por 5 vezes e as do lado de $60 \mathrm{~Hz}$ por 6 . A Figura 5 mostra, em destaque, todos os períodos de condução para as válvulas conectadas à fase A (no caso $T_{h_{1}}$ e $T_{h_{4}}$ ) do sistema de $50 \mathrm{~Hz}$, e a Figura 6, da mesma forma, para o lado de $60 \mathrm{~Hz}$. Em ambos os casos, as conduções para a tensão positiva correspondem a $T_{h_{1}}$ e para a tensão negativa a $T_{h_{4}}$. Observe que nessas figuras foi considerado ângulo de disparo de zero grau e é mostrado o intervalo de tempo referente a uma janela de $0,1 \mathrm{~s}$ (equivalente a um período para a freqüência de $10 \mathrm{~Hz}$ ).

Entretanto, é fácil notar que devido ao valor da nova freqüência fundamental, caso todos os harmônicos dessa freqüência sejam simulados, o sistema matricial dado em (1) apresentaria dimensões extremamente elevadas. Para contornar este problema, basta atentar para o fato de que apenas existirão no sistema, componentes harmônicos múltiplos inteiros de 50 ou $60 \mathrm{~Hz}$, não sendo possível a existência de nenhum outro

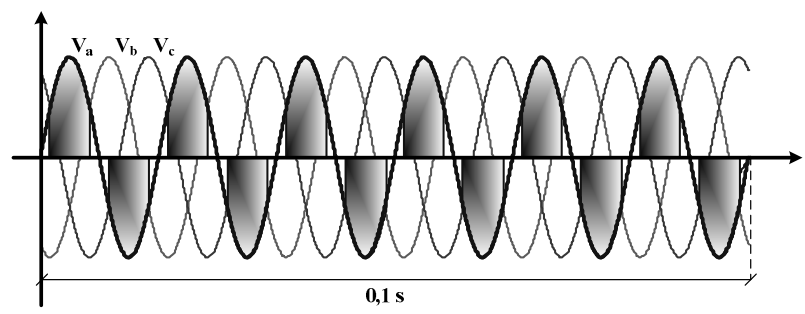

Figura 6: Períodos de Condução para as Válvulas do Lado de $60 \mathrm{~Hz}$.

componente. Assim, é suficiente simular apenas os múltiplos dessas duas frequiências $(0,50,60,100,120,150,180, \ldots)$ e ignorar todos os demais. Isso representa uma redução significativa das dimensões do sistema sem afetar, de nenhuma forma, os resultados.

\section{RESULTADOS}

Nesta seção são apresentados os resultados obtidos com a modelagem proposta. Inicialmente o modelo desenvolvido é validado com simulações comparativas com o ATP (Alternative Transients Program), no domínio do tempo. A seguir, com o intuito de verificar a robustez do modelo proposto para o HVDC, esse sistema é conectado à rede IEEE 14 Barras (IEEE, 1999), através da substituição do gerador conectado à barra 2 pelo HVDC. Todas as simulações foram realizadas com o sistema assíncrono, com o lado retificador operando a $50 \mathrm{~Hz}$ e o lado inversor a $60 \mathrm{~Hz}$ e a freqüência fundamental adotada foi de $10 \mathrm{~Hz}$. Todos os demais elementos do sistema, como linhas de transmissão, cargas, etc, são modelados de acordo com o apresentado em Variz (2006).

Para possibilitar uma melhor visualização dos gráficos, somente os componentes harmônicos com amplitude expressiva (superiores a $10^{-4} \mathrm{pu}$ ) são exibidos.

\subsection{Validação do Modelo}

Para a validação do modelo foi utilizado o sistema apresentado na Figura 7. Note que o sistema, apesar de pequeno, apresenta alguns complicadores, como a ausência de filtros (apenas o reator de alisamento é considerado) e poucas linhas de transmissão, o que favorecem as distorções harmônicas na rede e aumenta o grau de complexidade da simulação. Essa maior dificuldade nas simulações ocorre devido ao forte acoplamento harmônico existente nos conversores, onde a presença de um grande número de componentes harmônicos expressivos originalmente eliminados ou atenuados pelos filtros nos sistemas reais força a simulação de um número maior de componentes harmônicos, elevando a complexidade e a dimensão do sistema matemático. 


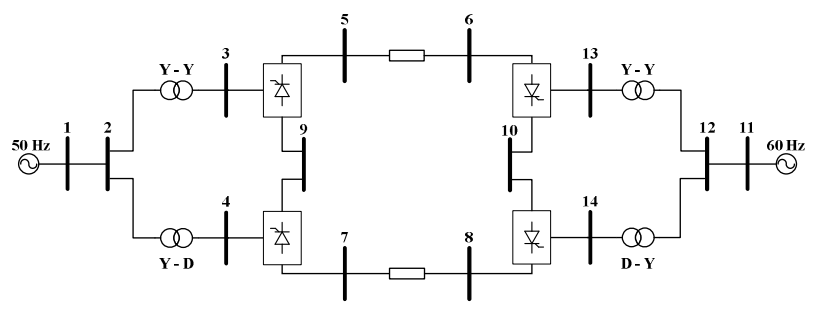

Figura 7: Rede Utilizada na Validação do Modelo: Sistema HVDC de 12 Pulsos.

Para as estações conversoras, os ângulos de disparo foram configurados em $15^{\circ}$ para o retificador $(\alpha)$ e $30^{\circ}$ para o inversor $(\beta)$. Os parâmetros das impedâncias das válvulas (Figura 3) são iguais a $0,500 \mathrm{pu}$ para a resistência $(R)$ e $0,005 \mathrm{pu}$ para a indutância $(L)$. O valor atribuído a essa indutância se mostra essencial para o problema, controlando as transições de corrente durante a comutação. Empiricamente verificase que valores muito baixos resultam em ondas de corrente do lado CA com grandes sobre-sinais nas transições entre os instantes de condução e não condução. Observa-se também que quando seu valor é elevado ocorre uma significativa redução da corrente $\mathrm{CC}$ e às vezes até mesmo problemas de convergência. Por isso é preciso cuidado na definição desse parâmetro. $\mathrm{O}$ valor do reator de alisamento foi incorporado à impedância da linha CC cujo valor total, para uma freqüência de $60 \mathrm{~Hz}$, é de $(0,046990+\mathrm{j} 13,8578) \mathrm{pu}$. Os transformadores utilizados foram dimensionados para ter a mesma impedância na freqüência nominal de cada sistema, com reatância de dispersão igual a 0,12601 pu e resistência série igual a $2 e^{-4}$ pu. O ramo magnetizante foi desconsiderado.

Devido a questões numéricas nas simulações do sistema no ATP, foi necessária a inclusão das linhas de transmissão entre as barras 1 e 2 , e entre 11 e 12 representadas por pequenas indutâncias em paralelo com grandes resistências, a saber, 2000 pu para as resistências e 0,0377 pu para as reatâncias (referidas a $60 \mathrm{~Hz}$ ).

Foram simulados todos os harmônicos múltiplos de 50 e 60 $\mathrm{Hz}$ até $2940 \mathrm{~Hz}$ (harmônico 49 de $60 \mathrm{~Hz}$ ), além do nível CC (frequiência zero), totalizando 91 freqüências harmônicas. $\mathrm{O}$ sistema total possui então, dimensão de $8190 \times 8190$, Apesar do tamanho elevado, o sistema é bastante esparso, o que garante uma boa agilidade no processo de solução.

A seguir são mostrados os gráficos comparativos para a validação do modelo proposto. Na Figura 8 é mostrado o espectro da corrente na fase $A$ para a barra 14 do inversor (barra CA) e em seguida, na Figura 9, o espectro da tensão de linha na mesma barra. Para melhorar a interpretação do gráfico, os componentes referentes a $60 \mathrm{~Hz}$ (nominal) foram suprimidos. Os valores deste componente $(60 \mathrm{~Hz})$ obtidos

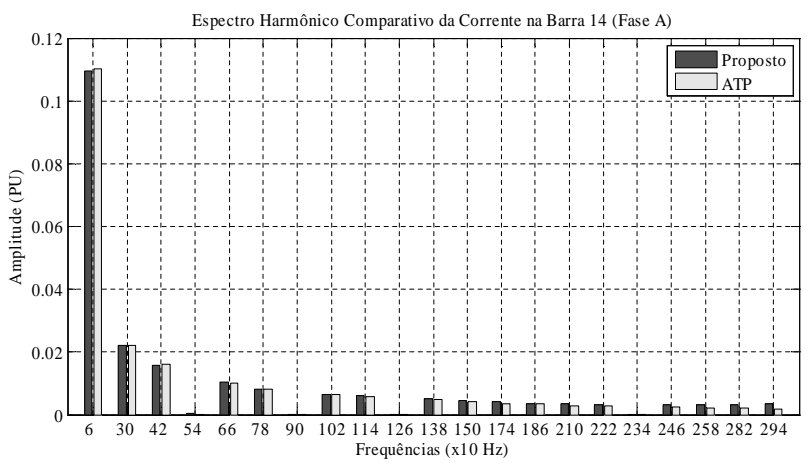

Figura 8: Espectro Comparativo da Corrente na Barra CA do Inversor (Barra 14).

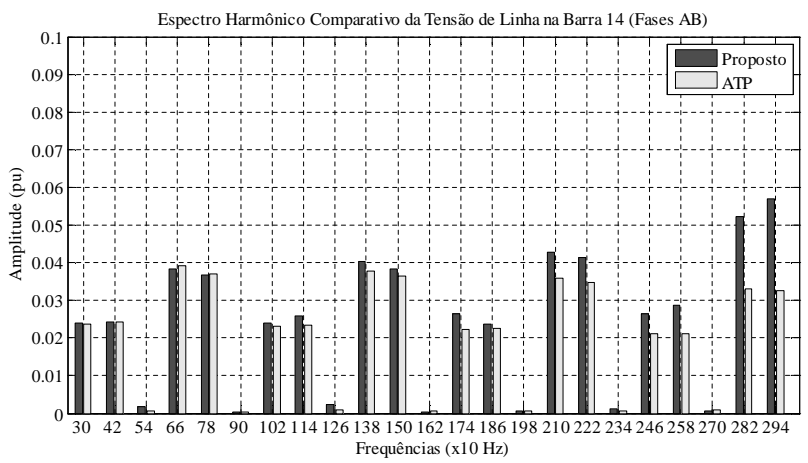

Figura 9: Espectro Comparativo da Tensão na Barra CA do Inversor (Barra 14).

pela metodologia proposta e pelo ATP foram 1,7131 pu e $1,7142 \mathrm{pu}$, respectivamente. Observe que as diferenças entre as simulações comparativas são mínimas. Note também que para facilitar a visualização e análise dos resultados, somente as freqüências com componente harmônico significativo são exibidas, fazendo com que o eixo das freqüências não esteja em escala.

É possível perceber em ambos os gráficos acima que apenas os harmônicos de ordem $6 n \pm 1$ (em relação a $60 \mathrm{~Hz}$ ) estão presentes. Isso ocorre porque a análise foi feita na barra CA de um dos conversores de 6 pulsos. Por outro lado, se a análise for feita na barra 12 que representa a junção CA dos inversores, ou seja, terminal CA da estação inversora (12 pulsos), somente haverá componentes harmônicos expressivos para as ordens $12 n \pm 1$. Isso pode ser verificado no espectro da tensão de linha das tensões no primário dos transformadores conectados ao inversor, apresentado na Figura 10. Novamente os componentes de $60 \mathrm{~Hz}$ são suprimidos, cujos valores são 1,7249 pu para a metodologia proposta e $1,7252 \mathrm{pu}$ para o ATP. Note que há uma atenuação do conteúdo harmônico nesta barra comparada ao da barra 14 (Figura 9), devido à presença dos transformadores de conexão. Graficamente é 


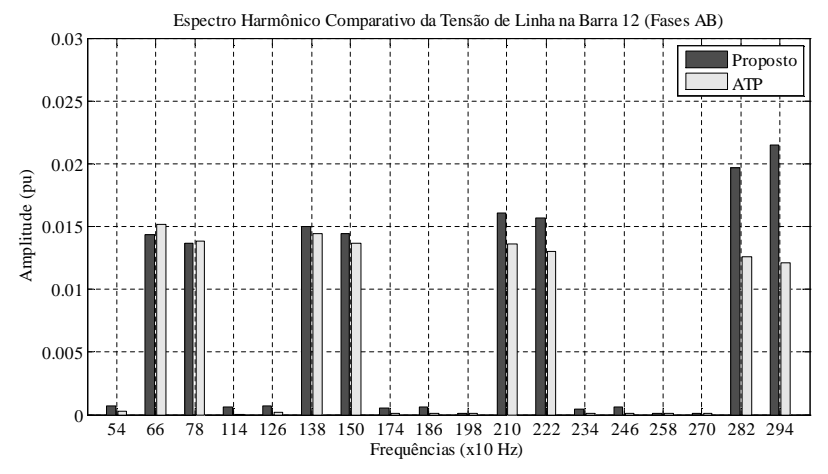

Figura 10: Espectro Harmônico Comparativo na Barra CA de Interface do Inversor (Primário dos Transformadores).

possível perceber que há uma maior divergência entre os resultados para as frequiências de maior valor. Isto ocorre pelo fato desta freqüência ser a última simulada pelo MICTH, portanto não possui a contribuição dos acoplamentos harmônicos com as ordens superiores. Isso é comprovado ao repetir a simulação considerando um maior número de harmônicos, onde essa divergência é novamente deslocada para o último componente simulado. Importante salientar que não ocorrem alterações significativas nos resultados para os componentes inferiores. E, como as freqüências mais prejudiciais para o sistema e para as cargas de forma geral são aquelas inferiores a $5 \mathrm{kHz}$ (IEEE, 1983), considera-se que os resultados obtidos, desconsiderando-se esse último componente, são satisfatórios.

Na Figura 11 são mostradas as formas de onda das tensões resultantes desses espectros, nas barras 14 e 12 . As oscilações presentes nas ondas da barra 14 referem-se aos chaveamentos entre válvulas, chamados notches.

A seguir, na Tabela 1, é mostrado o espectro comparativo da tensão e da corrente no sistema CC.

Observe que o espectro harmônico de corrente apresenta componentes de magnitude significativa somente para os componentes característicos $(12 n)$, que para o sistema corresponde aos múltiplos de 60 e 72 (referenciado a $10 \mathrm{~Hz}$ ). Note também que, diferentemente dos resultados anteriormente mostrados, ocorreram discrepâncias maiores entre as simulações, sem, no entanto, invalidar a formulação, pois parte disso se deve às diferenças inerentes aos controles de disparo dos tiristores nas duas plataformas de simulação.

\subsection{Sistema IEEE 14 Barras}

O mesmo sistema HVDC da seção anterior é agora inserido na rede IEEE 14 barras (IEEE, 1999) modificada, como mostrado na Figura 12. O gerador originalmente conectado à
Tabela 1: Espectro Harmônico Comparativo do Lado CC.

\begin{tabular}{|c|c|c|c|c|}
\hline $\begin{array}{c}\text { Freq } \\
(\mathrm{Hz})\end{array}$ & \multicolumn{2}{|c|}{ Corrente na Barra 5} & \multicolumn{2}{|c|}{ Tensão entre as Barras } \\
\hline & $\begin{array}{c}\text { Proposto } \\
(\mathrm{pu})\end{array}$ & ATP $(\mathrm{pu})$ & $\begin{array}{c}\text { Proposto } \\
(\mathrm{pu})\end{array}$ & ATP $(\mathrm{pu})$ \\
\hline 0 & 0,0993527 & 0,1003695 & 2,9926069 & 3,0249515 \\
\hline 120 & 0,0000179 & 0,0000128 & 0,0014848 & 0,0006045 \\
\hline 240 & 0,0000048 & 0,0000086 & 0,0008781 & 0,0004225 \\
\hline 360 & 0,0000024 & 0,0000078 & 0,0007612 & 0,0003144 \\
\hline 480 & 0,0000019 & 0,0000096 & 0,0008862 & 0,0003290 \\
\hline 600 & 0,0010801 & 0,0005256 & 0,0219992 & 0,0046432 \\
\hline 720 & 0,0009347 & 0,0007587 & 0,3273883 & 0,2592443 \\
\hline 840 & 0,0000028 & 0,0000040 & 0,0014581 & 0,0007125 \\
\hline 960 & 0,0000010 & 0,0000044 & 0,0008743 & 0,0003389 \\
\hline 1080 & 0,0000006 & 0,0000024 & 0,0007648 & 0,0002744 \\
\hline 1200 & 0,0004437 & 0,0001026 & 0,0181959 & 0,0018842 \\
\hline 1320 & 0,0000031 & 0,0000032 & 0,0014321 & 0,0005829 \\
\hline 1440 & 0,0003610 & 0,0001715 & 0,2531428 & 0,1168341 \\
\hline 1560 & 0,0000013 & 0,0000006 & 0,0014387 & 0,0006845 \\
\hline 1680 & 0,0000004 & 0,0000023 & 0,0008662 & 0,0003132 \\
\hline 1800 & 0,0002781 & 0,0000245 & 0,0171095 & 0,0008174 \\
\hline 1920 & 0,0000015 & 0,0000013 & 0,0008041 & 0,0002751 \\
\hline 2040 & 0,0000019 & 0,0000017 & 0,0014254 & 0,0004493 \\
\hline 2160 & 0,0002218 & 0,0000645 & 0,2329068 & 0,0659102 \\
\hline 2280 & 0,0000008 & 0,0000011 & 0,0013992 & 0,0006330 \\
\hline 2400 & 0,0001930 & 0,0000040 & 0,0158393 & 0,0002073 \\
\hline 2520 & 0,0000010 & 0,0000013 & 0,0006597 & 0,0002183 \\
\hline 2640 & 0,0000010 & 0,0000012 & 0,0007773 & 0,0002155 \\
\hline 2760 & 0,0000013 & 0,0000010 & 0,0013543 & 0,0003306 \\
\hline 2880 & 0,0001503 & 0,0000269 & 0,2105119 & 0,0378812 \\
\hline
\end{tabular}

barra 2 passa então a ter sua potência gerada transmitida para a rede através do elo CC. Os parâmetros do elo são os mesmos utilizados na etapa de validação. O sistema IEEE 14 barras utilizado tem todos os componentes representados como elementos trifásicos e a rede é desbalanceada com a utilização de cargas desequilibradas. Os parâmetros da rede são listados no apêndice. Note que a linha tracejada na Figura 12 é inserida apenas para facilitar a visualização do sistema unifilar simulado, não representando, portanto, uma linha de transmissão.

As simulações foram realizadas considerando todos os harmônicos múltiplos de 50 e $60 \mathrm{~Hz}$ até a frequiência de 4200 

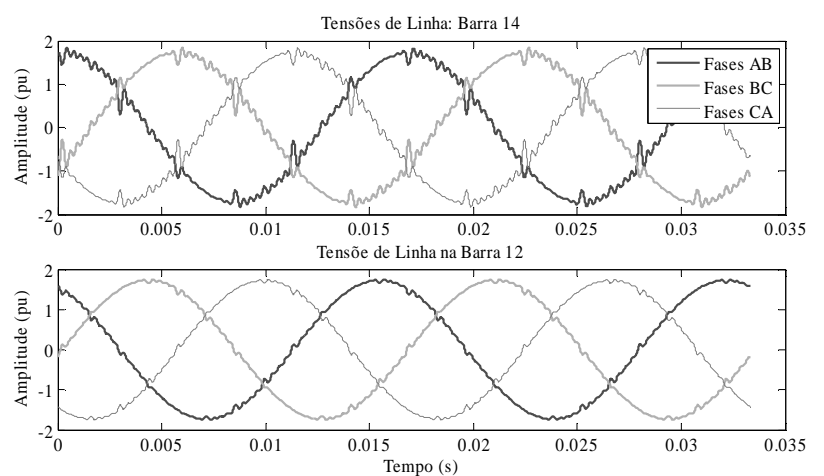

Figura 11: Formas de Onda das Tensões de Linha nas Barras do Inversor.
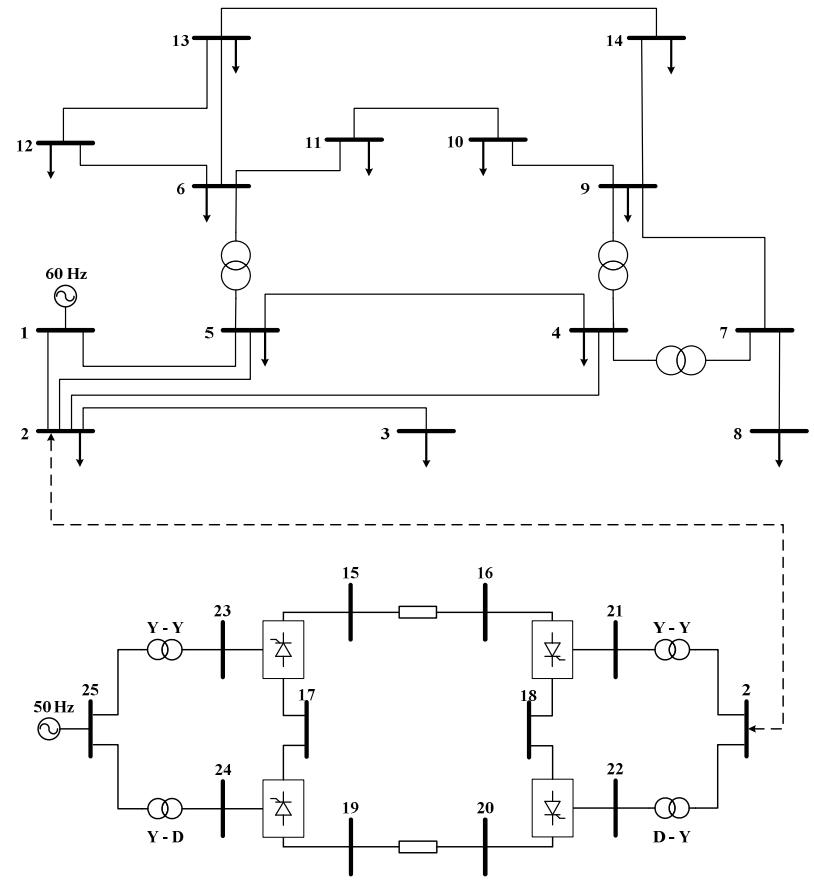

Figura 12: Sistema HVDC de 12 Pulsos Conectado à Rede IEEE 14 Barras.

$\mathrm{Hz}$ (harmônico 70 de $60 \mathrm{~Hz}$ ), totalizando 141 freqüências harmônicas. Apesar da grande dimensão, o sistema matricial é altamente esparso, com 98,9\% de elementos nulos.

Nas Figuras 13 e 14 é possível observar as formas de onda das correntes nos conversores. A Figura 13 mostra as correntes CA no inversor (barra 21) no ponto entre o conversor e o transformador. Observe que nesse ponto não é possível perceber os efeitos dos desequilíbrios, mesmo para o inversor. O espectro dessas ondas é mostrado na Figura 15, onde o componente de $60 \mathrm{~Hz}$, cujo valor é igual a 0,2144 pu para a fase $A, 0,2154$ pu para a fase $B$ e 0,2147 pu para a $C$, foi suprimido do gráfico. Na Figura 14 são mostradas as formas

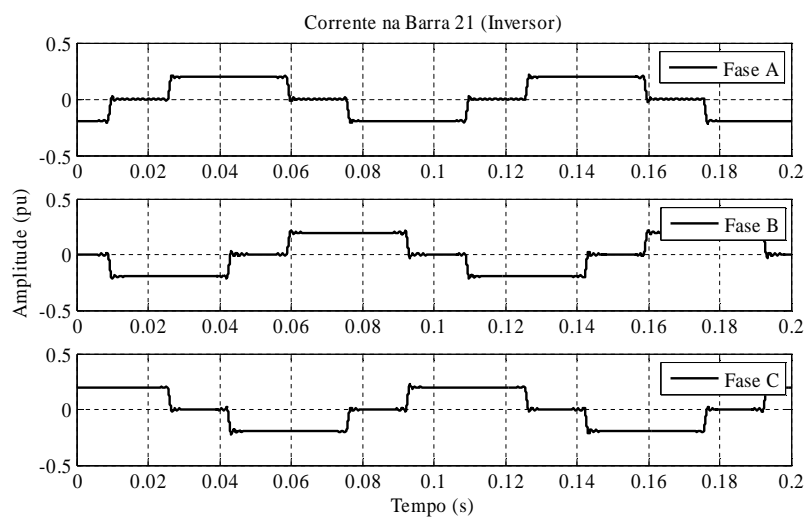

Figura 13: Corrente na Barra 21 do Inversor.

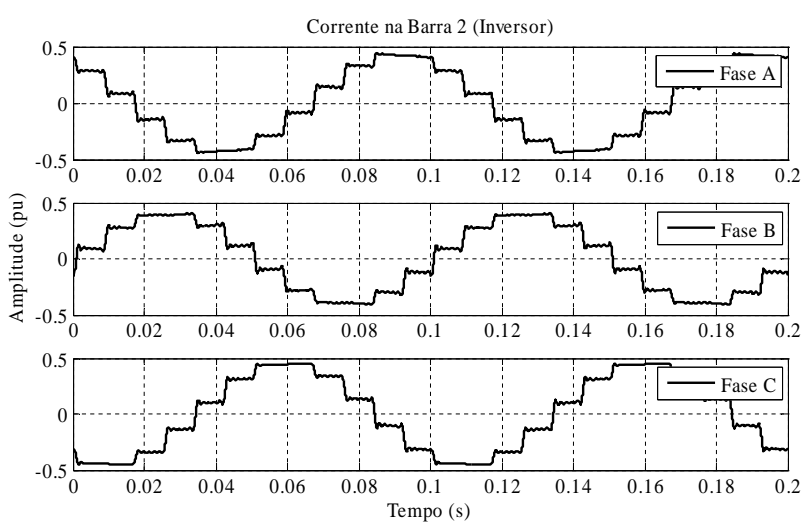

Figura 14: Corrente Injetada pelo HVDC na Rede CA (Barra 2).

de onda da corrente no ponto de conexão com a rede CA. É preciso salientar que as oscilações observadas nas bordas das formas de onda das correntes (apresentadas, por exemplo, na Figura 13) são intrínsecas da Série de Fourier e são conhecidas como Efeito de Gibbs (Proakis e Monolakis, 1996). Tais oscilações são independentes do número de harmônicos simulados e a amplitude do sobre-sinal, para ondas quadradas, será sempre igual a $9 \%$ do sinal.

Na Figura 16, é mostrado o espectro da corrente que flui pela linha CC. Observe que o reator de alisamento praticamente elimina os componentes harmônicos dessa corrente, deixando a corrente praticamente sem oscilações. Note que ocorre o surgimento, apesar de pequena magnitude, do componente de $2^{a}$ harmônico para o lado de $60 \mathrm{~Hz}$, correspondente a $120 \mathrm{~Hz}$.

A Figura 17 mostra a DHT das tensões de fase para todas as barras da rede CA. Nota-se claramente que o maior nível de distorção ocorre para a fase $C$ (à exceção da barra 3), uma vez que é a fase com menor impedância de carga. 


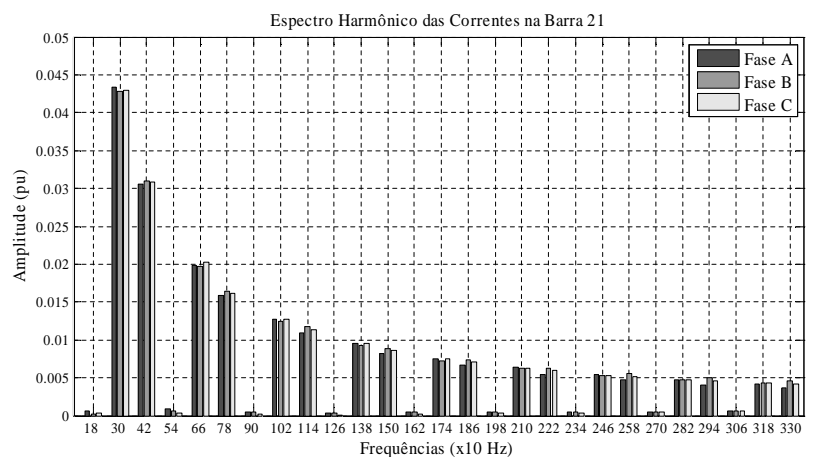

Figura 15: Corrente CA em um dos Pólos do Inversor.
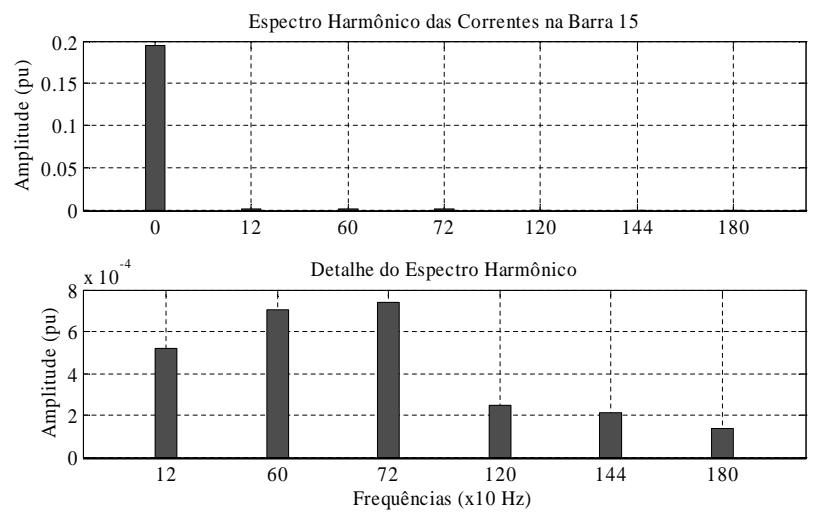

Figura 16: Espectro da Corrente CC do HVDC.

Na Tabela 2 é mostrado o espectro trifásico das tensões de linha nas barras 2 e 3 da rede $\mathrm{CA}$, onde o efeito dos desequilíbrios de carga é evidenciado.

Por fim, na Tabela 3, são expostos os fluxos de potência resultantes no sistema HVDC. Notam-se os efeitos dos desequilíbrios, bem como a característica indutiva do elo, consumindo reativos da rede na freqüência nominal do sistema.

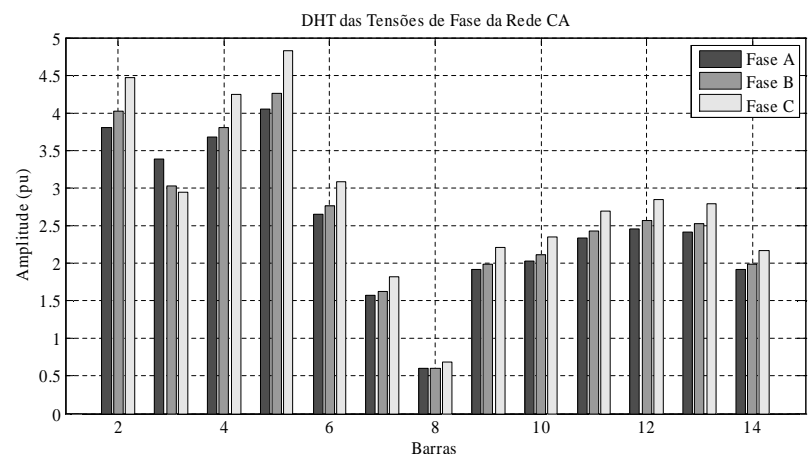

Figura 17: Distorção Harmônica Provocada pelo HVDC nas Tensões da Rede CA.
Tabela 2: Espectro Harmônico das Tensões de Linha nas Barras 2 e $3(\mathrm{pu})$.

\begin{tabular}{|c|c|c|c|c|c|c|}
\hline $\begin{array}{c}\text { Freq } \\
\text { (Hz) }\end{array}$ & \multicolumn{3}{|c|}{ Barra 2 } & \multicolumn{3}{|c|}{ Barra 3} \\
\hline & Fase A & Fase B & Fase C & Fase A & Fase B & Fase C \\
\hline 60 & 1,7622 & 1,753523 & 1,746081 & 1,714756 & 1,697024 & 1,663552 \\
\hline 180 & 0,000116 & 0,000105 & 0,000113 & 0,000106 & $9,38 \mathrm{e}-05$ & $9,69 \mathrm{e}-05$ \\
\hline 540 & 0,001184 & 0,001173 & 0,001091 & 0,00104 & 0,000888 & 0,000952 \\
\hline 660 & 0,049823 & 0,056225 & 0,052558 & 0,043599 & 0,04087 & 0,042328 \\
\hline 780 & 0,043993 & 0,046941 & 0,041735 & 0,033727 & 0,030629 & 0,029657 \\
\hline 900 & 0,001226 & 0,001217 & 0,001153 & 0,000537 & 0,000457 & 0,000476 \\
\hline 1140 & 0,000437 & 0,000404 & 0,000431 & $7,07 \mathrm{e}-05$ & $5,6 \mathrm{e}-05$ & $6,09 \mathrm{e}-05$ \\
\hline 1260 & 0,000139 & 0,00053 & 0,000637 & $2,73 \mathrm{e}-05$ & $7,35 \mathrm{e}-05$ & $9,29 \mathrm{e}-05$ \\
\hline 1380 & 0,015085 & 0,014876 & 0,01538 & 0,002388 & 0,002113 & 0,002106 \\
\hline 1500 & 0,011186 & 0,011583 & 0,010689 & 0,001534 & 0,001444 & 0,001509 \\
\hline 1620 & 0,000432 & 0,000449 & 0,000423 & $5,39 \mathrm{e}-05$ & $5,15 \mathrm{e}-05$ & $5,45 \mathrm{e}-05$ \\
\hline 1980 & $8,7 \mathrm{e}-05$ & 0,000107 & 0,000107 & $7,74 \mathrm{e}-06$ & $8,94 \mathrm{e}-06$ & $8,51 \mathrm{e}-06$ \\
\hline 2100 & 0,004596 & 0,004528 & 0,004683 & 0,000331 & 0,000312 & 0,000304 \\
\hline 2220 & 0,004005 & 0,004251 & 0,003706 & 0,000238 & 0,000241 & 0,00023 \\
\hline 2340 & 0,000273 & 0,000334 & 0,000269 & $1,45 \mathrm{e}-05$ & $1,72 \mathrm{e}-05$ & $1,49 \mathrm{e}-05$ \\
\hline 2820 & 0,002376 & 0,002351 & 0,002392 & $9,31 \mathrm{e}-05$ & $9,02 \mathrm{e}-05$ & $8,69 \mathrm{e}-05$ \\
\hline 2940 & 0,002179 & 0,002366 & 0,00194 & $7,64 \mathrm{e}-05$ & $8,08 \mathrm{e}-05$ & $7,1 \mathrm{e}-05$ \\
\hline 3060 & 0,000272 & 0,000283 & 0,000259 & $8,84 \mathrm{e}-06$ & $8,97 \mathrm{e}-06$ & $8,75 \mathrm{e}-06$ \\
\hline 3540 & 0,001517 & 0,001519 & 0,001469 & $3,84 \mathrm{e}-05$ & $3,8 \mathrm{e}-05$ & $3,51 \mathrm{e}-05$ \\
\hline 3660 & 0,001407 & 0,001611 & 0,001166 & $3,26 \mathrm{e}-05$ & $3,67 \mathrm{e}-05$ & $2,81 \mathrm{e}-05$ \\
\hline 3780 & 0,000256 & 0,000279 & 0,000237 & $5,57 \mathrm{e}-06$ & $5,95 \mathrm{e}-06$ & $5,34 \mathrm{e}-06$ \\
\hline 4140 & 0,000137 & 0,000133 & 0,000131 & $2,55 \mathrm{e}-06$ & $2,45 \mathrm{e}-06$ & $2,33 \mathrm{e}-06$ \\
\hline
\end{tabular}

Tabela 3: Fluxo de Potência Injetada pelo Elo na Rede CA pela Barra $2(\mathrm{pu})$.

\begin{tabular}{|c|c|c|c|c|c|c|}
\hline $\begin{array}{c}\text { Freq } \\
\text { (Hz) }\end{array}$ & \multicolumn{5}{|c|}{ Barra 2 } \\
\hline & P (Fase A) & P (Fase B) & P (Fase C) & P (Fase A) & P (Fase B) & P (Fase C) \\
\hline 60 & 0,367801 & 0,360478 & 0,406016 & $-0,25356$ & $-0,20198$ & $-0,22242$ \\
\hline 660 & 0,001044 & 0,000995 & 0,001195 & 0,000499 & 0,000594 & 0,000691 \\
\hline 780 & 0,000729 & 0,000841 & 0,000876 & 0,000217 & 0,000218 & 0,000111 \\
\hline 1380 & $4,68 \mathrm{e}-06$ & $4,37 \mathrm{e}-06$ & $4,469 \mathrm{e}-06$ & $-0,00017$ & $-0,00016$ & $-0,00017$ \\
\hline 1500 & $1,65 \mathrm{e}-06$ & $1,950 \mathrm{e}-06$ & $1,782 \mathrm{e}-06$ & $-0,0001$ & $-0,00012$ & $-0,00011$ \\
\hline 2100 & $1,97 \mathrm{e}-07$ & $1,88 \mathrm{e}-07$ & $1,990 \mathrm{e}-07$ & $-3,487 \mathrm{e}-05$ & $-3,258 \mathrm{e}-05$ & $-3,338 \mathrm{e}-05$ \\
\hline 2220 & $9,96 \mathrm{e}-08$ & $1,33 \mathrm{e}-07$ & $1,188 \mathrm{e}-07$ & $-2,382 \mathrm{e}-05$ & $-3,129 \mathrm{e}-05$ & $-2,731 \mathrm{e}-05$ \\
\hline
\end{tabular}




\section{CONCLUSÕES}

O desenvolvimento do modelo proposto para o HVDC baseado no método de injeção de corrente trifásico harmônico permitiu a implementação de uma nova ferramenta matemática e computacional para análise harmônica capaz de simular com eficiência e robustez, em regime permanente, sistemas elétricos de potência CA trifásicos com rede $\mathrm{CC}$ incorporada. Entretanto, sem as limitações geralmente encontradas nas demais metodologias no domínio da freqüência.

A validação do modelo proposto foi realizada através de simulações comparativas com o ATP (Alternative Transients Program). Apesar das metodologias serem totalmente distintas (domínio da frequiência $\times$ tempo), apenas pequenas diferenças foram observadas entre os resultados, mostrando a real viabilidade e precisão do modelo. A robustez do método pode ser comprovada pelos resultados apresentados, onde todos os harmônicos característicos gerados pela operação dos conversores do HVDC são corretamente calculados, mesmo sendo inicializados com valores nulos (somente a componente fundamental é inicializada adequadamente com valores de tensão de 1 pu. Apenas o último componente simulado deve ser desconsiderado, uma vez que este não possui as contribuições dos acoplamentos com as freqüências superiores.

A metodologia empregada se mostrou bastante versátil, permitindo simulação sistêmica e unificada de todos os componentes do sistema, inclusive da conexão CC. Os desequilíbrios puderam ser avaliados sem qualquer dificuldade adicional, através de representações reais de todos os componentes envolvidos. Além disso, a ferramenta desenvolvida permite a simulação de qualquer componente espectral, inclusive de componentes interharmônicos e subharmônicos, através da simples mudança na freqüência fundamental de simulação. Essa versatilidade na escolha das frequiências também poderia ser utilizada, por exemplo, para investigação do comportamento do sistema frente à presença de interharmônicos, produzidos por fornos a arco.

Apesar de não discutido no presente trabalho, existem diversas outras possibilidades de simulações utilizando o modelo de HVDC proposto sem a necessidade de alterações complexas na sua formulação, dentre os quais podemos listar:

- Representação de sistemas CCC-HVDC, que consistem de um aperfeiçoamento dos CSC-HVDC conseguido através da associação série de capacitores com os conversores (Ottosson e Kjellin, 2001; Sood, 2004);

- Simulação de sistemas back-to-back, ou seja, sem a presença de uma linha de transmissão CC;

- Modelagem de sistemas VSC-HVDC com utilização de chaves autocomutadas;

- Simulação de compensadores de energia reativa, como os STATCOM.

O modelo desenvolvido em conjunto com a metodologia utilizada apresentou resultados bastante promissores, com diversos campos de aplicação, e constitui uma importante contribuição para a análise de sistemas elétricos de potência em regime permanente.

\section{APÊNDICE}

Nesse apêndice são apresentados os parâmetros utilizados nas simulações. A rede teste IEEE 14 barras (IEEE, 1999; IEEE, 1973) foi adaptada para análise harmônica de sistemas trifásicos desbalanceados com presença de um elo CC com interconexão assíncrona. Os dados para as linhas de transmissão são apresentados na Tabela 4 (IEEE, 1999).

Tabela 4: Parâmetros das Linhas para o IEEE 14 Barras.

\begin{tabular}{|c|c|c|c|c|}
\hline De & Para & R $(\mathrm{pu})$ & $\mathrm{L}(\mathrm{pu})$ & Csh $(\mathrm{pu})$ \\
\hline 1 & 2 & 0,01938 & 0,000157 & 0,000140 \\
\hline 1 & 5 & 0,05403 & 0,000592 & 0,000131 \\
\hline 2 & 3 & 0,04699 & 0,000525 & 0,000116 \\
\hline 2 & 4 & 0,05811 & 0,000468 & $9,02 \mathrm{e}-05$ \\
\hline 2 & 5 & 0,05695 & 0,000461 & $9,18 \mathrm{e}-05$ \\
\hline 3 & 4 & 0,06701 & 0,000454 & $3,40 \mathrm{e}-05$ \\
\hline 4 & 5 & 0,01335 & 0,000112 & - \\
\hline 6 & 11 & 0,09498 & 0,000528 & - \\
\hline 6 & 12 & 0,12291 & 0,000679 & - \\
\hline 6 & 13 & 0,06615 & 0,000346 & - \\
\hline 7 & 8 & $1,00 \mathrm{e}-10$ & 0,000467 & - \\
\hline 7 & 9 & $1,00 \mathrm{e}-10$ & 0,000292 & - \\
\hline 9 & 10 & 0,03181 & 0,000224 & - \\
\hline 9 & 14 & 0,12711 & 0,000717 & - \\
\hline 10 & 11 & 0,08205 & 0,000509 & - \\
\hline 12 & 13 & 0,22092 & 0,00053 & - \\
\hline 13 & 14 & 0,17093 & 0,000923 & - \\
\hline 15 & 16 & 0,46990 & 0,036759 & - \\
\hline 19 & 20 & 0,46990 & 0,036759 & - \\
\hline & & & & \\
\hline
\end{tabular}

Note que as linhas que conectam as barras 15 e 16 e as barras 19 e 20 são circuitos CC, cujos valores dos reatores de alisamento já estão incluídos no valor da indutância da linha. 
As cargas foram consideradas como sendo do tipo impedância constante e modeladas como associações de componentes de resistência e reatância em série ( $R L$ ou $R C$ série). Os parâmetros das cargas são listados na Tabela 5 . Note que para cargas conectadas em delta, os valores são referentes aos ramos entre as fases $A B, B C$ e $C A$.

Tabela 5: Cargas do Sistema IEEE 14 Barras.

\begin{tabular}{|c|c|c|c|c|c|c|c|}
\hline Barra & $\mathrm{R}(\mathrm{pu})$ & $\mathrm{L}(\mathrm{pu})$ & $\mathrm{C}(\mathrm{pu})$ & Conexão & $\begin{array}{c}\text { Fase } \\
\mathrm{A}\end{array}$ & $\begin{array}{c}\text { Fase } \\
\mathrm{B}\end{array}$ & $\begin{array}{c}\text { Fase } \\
\mathrm{C}\end{array}$ \\
\hline 2 & 3,748449 & 0,005819 & - & Estrela & $100 \%$ & $100 \%$ & $100 \%$ \\
\hline 3 & 1,080551 & - & 0,052556 & Estrela & $120 \%$ & $100 \%$ & $80 \%$ \\
\hline 4 & 6,473814 & - & 0,005022 & Delta & $100 \%$ & $100 \%$ & $100 \%$ \\
\hline 5 & 13,108488 & 0,007320 & - & Estrela & $120 \%$ & $100 \%$ & $80 \%$ \\
\hline 6 & 26,075130 & - & 0,000242 & Delta & $100 \%$ & $100 \%$ & $100 \%$ \\
\hline 8 & $1,00 \mathrm{e}-10$ & - & 0,000388 & Estrela & $100 \%$ & $100 \%$ & $100 \%$ \\
\hline 9 & 2,871027 & 0,004285 & - & Estrela & $100 \%$ & $100 \%$ & $100 \%$ \\
\hline 10 & 8,671850 & 0,014824 & - & Estrela & $120 \%$ & $100 \%$ & $80 \%$ \\
\hline 11 & 25,244490 & 0,034438 & - & Estrela & $100 \%$ & $100 \%$ & $100 \%$ \\
\hline 12 & 51,21538 & 0,035634 & - & Delta & $100 \%$ & $100 \%$ & $100 \%$ \\
\hline 13 & 6,894136 & 0,007857 & - & Estrela & $100 \%$ & $100 \%$ & $100 \%$ \\
\hline 14 & 19,42283 & 0,017289 & - & Delta & $100 \%$ & $100 \%$ & $100 \%$ \\
\hline
\end{tabular}

\section{AGRADECIMENTOS}

Os autores agradecem ao Programa de Pós-graduação em Engenharia Elétrica da UFJF (PPEE-UFJF), ao Programa de Pós-graduação em Engenharia da UFRJ (COPPE-UFRJ), e à CAPES. Este trabalho é beneficiário de auxílio financeiro da CAPES - Brasil.

\section{REFERÊNCIAS}

Agelidis, V. G.; Demetriades, G. D.; Flourentzou, N. (2006). Recent Advances in High-Voltage DirectCurrent Power Transmission Systems. IEEE International Conference on Industrial Technology, ICIT, pp. 206-213, 15-17 Dezembro.

Bathurst, G. N.; Smith, B. C.; Watson, N. R. e Arrillaga, J. (1999). Modelling of HVDC Transmission Systems in the Harmonic Domain. IEEE Transactions on Power Delivery, Vol. 14, No. 3; pp. 1075-1080, July 1999.

Dommel, H. W. (1986). Electromagnetic Transients Program Reference Manual (EMTP Theorycal Book), Prepared for Bonneville Power Administration, Dept. of Electrical Engineering, University of British Columbia.
Faruque, M. O.; Zhang, Y.; Dinavahi, V. (2006). Detailed Modeling of CIGRÉ HVDC Benchmark System Using PSCAD/EMTDC and PSB/SIMULINK. IEEE Transactions on Power Delivery, Vol. 21, No. 1, pp. 378387, Jan. 2006.

Graham, J.; Jonsson B.; Moni, R.S. (2002). The Garabi 2000 MW Interconnection Back-to-Back HVDC to Connect Weak AC Systems. ABB Utilities AB, SE-771 80 Ludvika, Sweden.

IEEE Working Group on a Common Format for the Exchange of Solved Load Flow Data (1973). Common Data Format for the Exchange of Solved Load Flow Data, IEEE Transactions on Power Apparatus and Systems_, Vol. PAS-92, No. 6, November/December 1973, pp. 1916-1925.

IEEE Working Group on Power System Harmonics (1983). Power System Harmonics: An Overview. IEEE Transactions on Power Apparatus and Systems, Vol. PAS102, No. 8, August 1983.

IEEE (1993). IEEE Recomemend Practices and Requirements for Harmonic Control in Electrical Power Systems, IEEE std. 519-1993.

IEEE Task Force on Harmonics Modeling and Simulation (1999). Test Systems for Harmonics Modeling and Simulation. IEEE Transactions on Power Delivery, Vol. 14, No. 2, April 1999.

IEEE Working Group on HVDC and FACTS Bibliography and Records (2006). DC and Flexible AC Transmission Subcommittee of the IEEE Transmission and Distribution Committee.

Kundur, P. (1994). Power System Stability and Control. McGraw-Hill.

Madrigal, M. e Acha, E., (2001). Harmonic Modelling of Source Converters for HVDC Stations, IEE AC-DC Power Transmission Conference, No. 485, pp. 125-131.

Niquini, F. M. M.; Variz, A. M.; Pereira, J. L. R.; Barbosa, P. G. e Carneiro Jr., S. (2008a). Frequency Domain Modeling of Monopolar HVDC Link Using ThreePhase Harmonic Current Injection Method, IEEE PES $T \& D$ Conference and Exposition Latin-America. August, 2008, Bogotá, Colômbia.

Niquini, F. M. M.; Variz, A. M.; Pereira, J. L. R.; Barbosa, P. G. e Carneiro Jr., S. (2008b). Modelagem do Elo de Corrente Contínua Monopolar no Domínio da Frequiência Utilizando o Método de Injeção de Correntes Trifásico Harmônico, XVII Congresso Brasileiro de Automática, CBA, Setembro de 2008, Juiz de Fora, Brasil. 
Niquini, F. M. M.; Variz, A. M.; Pereira, J. L. R.; Barbosa, P. G. e Carneiro Jr., S. (2009a). Modelagem do Elo de Corrente Contínua para Análise Harmônica Utilizando o Método de Injeção de Corrente Trifásico Harmônico com Solução Iterativa. XI Simpósio de Especialistas em Planejamento da Operação e Expansão Elétrica, SEPOPE, 16 a 20 de Março 2009, Belém, Brasil.

Niquini, F. M. M. (2009b). Modelagem do Elo de Corrente Contínua para Análise Harmônica Utilizando o Método de Injeção de Correntes Trifásico Harmônico com Solução Iterativa, Dissertação de Mestrado em Engenharia Elétrica, UFJF, Juiz de Fora, Brasil.

ONS (2007). Dados Relevantes 2007. Disponível em http: / / www.ons.org.br/download/ biblioteca_virtual/publicacoes/ dados_relevantes_2007.pdf. Página da Internet acessada em 08/12/2008.

Ottoson, N.; Kjellin, L. (r2001). Modular Back-toBack HVDC, with Capacitor Commutated Converters (CCC). IEE AC-DC Power Transmission, No 485, 2830 November 2001.

Praça, A. et alli (1996). Itaipu HVDC Transmission System 10 Years Operational Experience. V SEPOPE Recife, Brasil, 19 Maio.

Proakis, J. G., Monolakis, D. G. (1996). Digital Signal Processing: principles, algorithms, and applications. Ed. Prentice Hall.

Rudervall, R. et alli (2000). High Voltage Direct Current (HVDC) Transmission Systems Technology, Review Paper, Energy Week, Washington, D.C., USA, March 7-8.

Smith, B. C., Arrilaga, J., Wood, A. R. e Watson, N. R. (1995). Steady State Model of the AC/DC Convertor in the Harmonic Domain, IEE Proc.-Gener. Transm. Distrib., Vol. 142, No.2, p. 109-118, March 1995.

Sood, V. K. (2004). HVDC and FACTS Controllers Applications of Static Converters in Power Systems. Kluwer Academic Publishers, Boston, United States.

Variz, A. M. (2006). Cálculo do Fluxo de Harmônicas em Sistemas Trifásicos Utilizando o Método de Injeção de Correntes, Tese de D. Sc., Coppe/UFRJ, Rio de Janeiro.

Variz, A. M.; Pereira, J. L. R.; Carneiro Jr., S. e Barbosa, P. G. (2008). Cálculo do Fluxo de Harmônicos em Sistemas de Potência Trifásicos Utilizando o Método de Injeção de Correntes com Solução Iterativa, Revista SBA Controle \& Automação vol.19 no. 2, pp. 178-198, Abril, Maio e Junho 2008. 